\title{
Les mises en scène de la visite guidée.
}

\section{Communication et médiation}

De Michèle Gellereau - Paris, Editions L'Harmattan, coll. Communication et Civilisation, 2005, 279 p.

\section{Isabelle Cousserand}

\section{(2) OpenEdition}

\section{Journals}

Édition électronique

URL : http://journals.openedition.org/communicationorganisation/3501

DOI : 10.4000/communicationorganisation.3501

ISSN : 1775-3546

Éditeur

Presses universitaires de Bordeaux

Édition imprimée

Date de publication : 1 octobre 2006

ISSN : 1168-5549

Référence électronique

Isabelle Cousserand, "Les mises en scène de la visite guidée. Communication et médiation », Communication et organisation [En ligne], 30 | 2006, mis en ligne le 21 juin 2012, consulté le 21 septembre 2020. URL : http://journals.openedition.org/communicationorganisation/3501 ; DOI : https://doi.org/10.4000/communicationorganisation.3501

Ce document a été généré automatiquement le 21 septembre 2020

(c) Presses universitaires de Bordeaux 


\section{Les mises en scène de la visite guidée. Communication et médiation}

De Michèle Gellereau - Paris, Editions L'Harmattan, coll. Communication et Civilisation, 2005, 279 p.

Isabelle Cousserand

1 Lors du colloque « Non-verbal, communication, organisation » organisé par le GREC/O en mai 2000, Michèle Gellereau avait présenté un travail qui, selon ses propres termes, entrait alors " dans le cadre d'un projet en gestation sur l'accompagnement des visiteurs dans la visite des sites patrimoniaux $»^{1}$. Quelques années plus tard, elle nous livre l'aboutissement de sa réflexion dans un ouvrage préfacé par Jean Davallon. Là où l'approche muséale s'intéresse souvent aux dispositifs techniques ou aux technologies interactives, Michèle Gellereau nous propose une réflexion centrée sur un type de médiation singulier, la « muséologie de point de vue », qui, selon Jean Davallon, « (...) vise à construire un point de vue pour le visiteur, (...) est centrée sur le visiteur et travaille le "placement" (et le déplacement) de celui-ci ». Son travail s'inscrit dans une perspective élargie qui interroge la construction des identités collectives et les dispositifs de compréhension des autres dans l'espace public. Il s'appuie aussi bien sur les pratiques langagières de la médiation du guide, la mise en scène des visites, que les interactions.

2 Le projet nourri par Michèle Gellereau est d'étudier la visite comme mode de médiation culturelle spécifique et la construction du récit comme forme privilégiée de partage. L'auteur, qui s'inscrit dans une approche sémio-pragmatique ${ }^{3}$ des représentations patrimoniales, a opté pour une démarche à la fois observationnelle et compréhensive. Elle a donc suivi de nombreuses visites et a mené des entretiens avec l'ensemble des acteurs des musées, "dans le souci d'analyser à la fois le dispositif de communication, l'objet produit et les discours de la situation (...) »4. Elle renouvelle ainsi la façon d'appréhender le rapport entre exposition et médiation en s'appuyant sur la muséologie, l'anthropologie et la communication. ${ }^{5}$ 
3 L'ouvrage est bâti sur un mode ternaire. La première partie, "Scènes et acteurs, places et rôles", s'intéresse d'abord aux objectifs assignés à la visite guidée, aux spécificités (et aux contraintes) de la mise en scène, sans pour autant oublier la dimension des relations publiques. Puis sont envisagés le statut des guides, leurs formations, leurs compétences et leurs rôles. L'ensemble présente une analyse de la diversité des objectifs, des situations, des positions mais aussi des enjeux organisationnels.

4 La seconde partie intitulée "L'interprétation du guide : le récit comme mode de médiation " offre une réflexion sur la construction du récit de la visite. L'auteur s'interroge tout d'abord sur le sens du récit, ses dimensions énonciatives et discursives, puis elle pose les questions de l'interprétation et de l'appropriation, pour déboucher sur celles de l'intégration et de la transmission des valeurs qui relèvent aussi de l'induction. Il s'agit donc de voir comment le visiteur est engagé à partager une vision de l'univers qu'il découvre.

5 Dans la troisième partie, "Une communication dialogale: appropriation et partage", Michèle Gellereau " (...) s'intéresse à la prise en compte du visiteur dans le discours du guide et aux effets de celle-ci dans la construction des récits collectifs " ${ }^{6}$. Elle revient sur les dimensions verbales et non verbales des interactions (le rôle du corps, par exemple) qui sont orientées vers un partage et tiennent compte de l'interprétation du visiteur. Puis elle traite des différents aspects du dialogue et $\mathrm{du}$ rôle de ce dernier dans le développement des identités narratives qui mènent au récit pour le collectif, que l'auteur différencie du récit collectif.

6 Visites guidées d'entreprises et de sites patrimoniaux sont en expansion. Dans un contexte de diversification du public visiteur, il est essentiel d'éclairer le rôle de transmission et de médiation du guide, traditionnellement associé au champ de l'action culturelle, donc moins abordé dans une perspective communicationnelle et relationnelle. De fait, comme le rappelle Jean Davallon dans la préface, «(...) l'analyse de la visite guidée appelle à regarder d'un autre ceil la mise en exposition, car elle porte attention à des processus qui ne sont pas a priori faciles à saisir dans celle-ci $»^{7}$.

7 Ainsi, Michèle Gellereau positionne son travail au carrefour de plusieurs thèmes (espace public, identité collective, mémoire) et combine des approches complémentaires (narration et situations de communication). "Ce que montre la visite guidée, c'est toute la difficulté pour le médiateur culturel à créer un monde partagé autre que minimal, fusionnel et consensuel, facilement accessible à un public venu pour se distraire ou découvrir du merveilleux. $\|^{8}$

8 C'est un travail de recherche pluridisciplinaire mené et présenté avec rigueur et passion, qui s'appuie sur des exemples précis et démontre combien « (...) l'analyse de la visite guidée mérite d'être prise au sérieux pour avancer dans la compréhension de la fabrique collective du monde, dans les interactions communicatives produites dans l'action culturelle ${ }^{9}$. Le propos novateur et l'analyse étayée font de cette recherche un modèle pour ceux qui aspirent à l'exigence. L'ouvrage est l'un des dignes représentants d'une édition de qualité. 


\section{NOTES}

1. Michèle Gellereau, "Paroles et gestes dans la visite guidée ", in Non-verbal, communication, organisation, COMMUNICATION \& ORGANISATION $\mathrm{N}^{\circ} 18,2^{\mathrm{e}}$ semestre 2000, université Michel de Montaigne-Bordeaux 3, p. 339-349.

2. p. 10 .

3. «Mon approche est essentiellement sémio-pragmatique au double sens du terme: d'une part parce que je tente de comprendre le sens de productions en fonction de leur contexte et de leurs pratiques, mais aussi parce que je tente de construire des interprétations à partir de l'observation empirique de ces pratiques, voire de la pratique elle-même. " p. 25.

4. p. 25. "J'ai donc suivi le travail d'une centaine de guides (...) et réalisé des entretiens avec la majorité d'entre eux, ainsi qu'avec des directeurs de structures, conservateurs ou médiateurs dans des lieux relativement divers (...) ». p. 28.

5. "Jusqu'à présent, on avait plutôt coutume de considérer la visite guidée dans sa dimension de complément de la mise en exposition. C'est ainsi que l'usage habituel de la notion de "médiation" laisse entendre que celle-ci est, soit une mise en rapport des visiteurs avec une cuvre, soit au contraire une explicitation de la mise en exposition. La première modalité apparaît plutôt comme l'apanage du musée ou de l'exposition d'art : son principe affiché de faciliter l'accès du visiteur à l'œuvre d'art va dans ce sens. La seconde se rencontre de préférence dans les expositions ou les musées de sciences : que la mise en exposition soit au service d'une compréhension des savoirs par les visiteurs n'est probablement pas étrangère au fait que la médiation reprenne, accompagne, complète et parfois même vienne se substituer partiellement à la mise en exposition. Ces types de muséologie, centrée sur l'objet dans un cas et sur le savoir dans l'autre, appelleraient en quelque sorte leurs types de médiation. Sans aller contre ce constat élémentaire, une analyse détaillée de la visite guidée va cependant à l'encontre d'un tel partage convenu entre mise en exposition et médiation. ", préface de Jean Davallon, p. 12.

6. p. 31 .

7. p. 12 .

8. p. 256.

9. p. 265 . 